\title{
Influencia de una cultura organizativa orientada hacia la calidad en los beneficios de la implantación de un sistema de gestión de recursos ERP
}

\author{
Oscar F. Bustinza, Ma Nieves Perez-Arostegui, Antonia Ruiz-Moreno \\ Universidad de Granada (Spain) \\ oscarfb@ugr.es, mnperez@ugr.es, aruizmor@ugr.es
}

Recibido: Enero 2013

Aceptado: Febrero 2013

\section{Resumen}

Objeto: La cultura organizativa orientada hacia la calidad tiene como objetivo satisfacer las necesidades del cliente, potenciar el trabajo en equipo, y orientar los procesos hacia una dinámica de mejora continua. El objetivo de este trabajo es analizar si, efectivamente, la cultura orientada hacia la calidad produce un efecto en la gestión de los procesos de la empresa, para la que se analiza su relación con los beneficios de un sistema de gestión de recursos o ERP.

Diseño/metodología/enfoque: Se configura un cuestionario con las principales variables del modelo y se procede a la realización de una encuesta entre empresas españolas. Se obtienen más de 200 cuestionarios válidos y se analiza el modelo mediante un sistema de ecuaciones estructurales.

Aportaciones $y$ resultados: Los resultados permiten afirmar que el compromiso de la empresa hacia la gestión de la calidad, el enfoque hacia el cliente, y la confianza de los trabajadores repercute positivamente en los resultados de tipo operativo, directivo y estratégico derivados la implantación de un sistema ERP. Sin embargo, no se encuentra relación entre el enfoque hacia el cliente y los beneficios organizativos, o los derivados de una mayor capacidad del sistema.

Originalidad/valor añadido: El presente trabajo permite analizar la relación entre la cultura orientada hacia la calidad por parte de la empresa y los sistemas de gestión de recursos clarificando sus aspectos positivos y señalando sus limitaciones. En este 
sentido, la orientación hacia el cliente podría limitar la flexibilidad de negocio al sustraer una gran cantidad de recursos, y generar disconformidad en los trabajadores derivada de la atención a las reclamaciones del cliente.

Palabras clave: Calidad, cultura organizativa, beneficios ERP

Código JEL: M10 - General; M14 - Cultura corporativa

Title: Organizational culture focused on quality management and benefits derived from an ERP system implementation

\section{Abstract}

Purpose: Organizational culture focused on quality management aims to meet customer needs and enhance teamwork, being oriented toward a dynamic process of continuous improvement. The purpose of this paper is to analyze whether, indeed, the qualityoriented culture has an effect on the management of business processes. In doing so, we analyze their relationship with the benefits of a resource management system or ERP.

Design/methodology/approach: A survey is used to collect data, with valid questionnaires obtained for 200 Spain based respondents. Empirical analysis utilises Structural Equation Modelling (SEM).

Findings: The results confirm that firm's commitment with quality management, customer focus, and confidence of workers has a positive effect on the results of operational, strategic and managerial benefits derived from an ERP system implementation. However, there is not relationship between customer focus and organizational benefits, neither to increase system capacity.

Originality/value: The present study analyzes the relationship between qualityoriented culture and the resource management systems of the firm clarifying their strengths and limitations. In this sense, the customer orientation may limit the flexibility of business as require a lot of resources, and generate dissatisfaction among workers resulting from the attention to customer complaints.

Keywords: Quality, organizational culture, ERP benefits

JEL Codes: M10 - General; M14 - Corporative culture 


\section{Introducción}

Los sistemas de gestión y asignación de recursos Enterprise Resource Planning (ERP) constituyen una de las herramientas esenciales en la gestión de las operaciones diarias de la empresa. Su objetivo es automatizar aquellos procesos de negocio que tengan como soporte el tratamiento de datos. El desarrollo de este tipo de sistemas ha sido creciente y se engloban en lo que se denomina Sistemas Avanzados de Gestión Empresarial, que tratan de manejar los sistemas de información de la empresa relacionando las distintas actividades de producción y suministro, ofreciendo diferentes indicadores útiles para analizar la gestión diaria de la empresa (Stadtler \& Kilger, 2008). Estos sistemas se utilizan para conseguir una mejora continua en los productos y servicios ofrecidos al cliente ( $\mathrm{Ng}$, Ip \& Lee, 1999), y contribuyen a mejorar la posición competitiva de la empresa al facilitar el acceso y tratamiento de la información de la que dispone, siendo esta información una de las fuentes principales de obtención de ventajas competitivas sostenibles (Arana-Solares, Alfalla-Luque \& Machuca, 2012).

Por otro lado, existen marcos globales de gestión en la empresas que buscan obtener ventajas mediante diferenciación de productos, compitiendo básicamente con el concepto de gestión de la calidad (Ruíz-Guerra, Martín-López \& Molina-Moreno, 2012). Este tipo de gestión se entiende como un compromiso hacia una cultura organizativa orientada hacia la calidad abarcando todos los procesos desarrollados por la empresa (Dean \& Evans, 1994). La gestión de la calidad está basada en una serie de principios, prácticas y técnicas focalizadas en satisfacer las necesidades del cliente, potenciar el trabajo en equipo, y orientar los procesos hacia una dinámica de mejora continua. Es en este último punto donde la reingeniería de procesos juega un papel determinante, siendo necesario considerar el papel que los sistemas ERP tienen en dicha reconfiguración al alinear la asignación de recursos a los objetivos de producción. La revisión de la literatura señala que existen beneficios potenciales y prácticos de un sistema orientado hacia la calidad (Schniederjans \& Kim, 2003; Jones, Cline \& Ryan, 2006), pero no ha quedado debidamente constatada la influencia que la cultura orientada hacia la calidad tiene sobre la gestión de los recursos en la empresa. Entendiendo que uno de los objetivos de la gestión de la calidad es la mejora continua de las operaciones de la empresa, debe existir un compromiso directivo a la hora de examinar de manera constante las técnicas y procesos de asignación de recursos en busca de los métodos más adecuados. Este es el planteamiento y la pregunta de investigación del presente trabajo, analizar la influencia de una cultura organizativa orientada hacia la calidad en los beneficios de la implantación de un sistema de gestión de recursos ERP, partiendo de la necesidad de cubrir una carencia de la literatura acerca de los beneficios prácticos del compromiso con la calidad en las organizaciones.

Para dar respuesta a la pregunta de investigación se procede a analizar una muestra de empresas en España que asumen una cultura organizativa orientada hacia la calidad en sus 
procesos y que, a su vez, tienen implantado un sistema de gestión de recursos ERP, realizando un análisis empírico de las relaciones propuestas. Para ello, se plantearan los fundamentos teóricos que sustentan el trabajo, se establecerá un modelo de ecuaciones estructurales, se recogerá el conjunto de hipótesis planteadas, se analizarán los resultados obtenidos y, finalmente, se expondrán las conclusiones alcanzadas.

\section{Revisión de la literatura}

\section{Cultura organizativa orientada hacia la gestión de la calidad}

Una cultura orientada a los principios que promueve la calidad constituye un elemento necesario para la permanencia y consolidación en la organización de sistemas de gestión de la calidad. Distintos estudios han demostrado que la cultura organizativa de apoyo a la gestión de la calidad es necesaria para conseguir el éxito de la implantación de sistemas basados en esos principios (Hackman \& Wageman, 1995; Chung, Hsu \& Tsai, 2010). Esta cultura ha sido estudiada de distintas formas y alternativas, pero una definición aceptada por la comunidad científica es aquella que la entiende como un sistema formado por un conjunto de valores, símbolos, rituales, mitos y prácticas que han evolucionado con el tiempo y que determinan la visión compartida por los miembros de la organización (Schein, 2004; Suppiah \& Sandhu, 2011).

En la literatura encontramos distintos trabajos que tratan de clarificar los límites de la cultura organizacional enfocada en la calidad. Autores como Zeitz, Johannesson y Ritchie (1997) o Adebanjo y Kehoe (1998) proponen distintas dimensiones de la cultura de calidad entre las que destacan: la comunicación, confianza entre la alta dirección y los empleados, el liderazgo de dirección o la orientación al cliente. Por tanto, el inicio de este camino hacia una cultura organizativa que apoye la calidad debe comenzar con el liderazgo y compromiso de la dirección con la calidad (Powell, 1995; Samson \& Terziovski, 1999; Foster, Horward \& Shannon, 2002; Sureshchandar, Rajemdran \& Anantharaman, 2003; Douglas \& Fredendall, 2004; Lee, Cheng, Yeung \& Lai, 2011). Tanto los precursores de la calidad como los distintos modelos conceptuales de la implantación de la misma consideran este compromiso esencial para el éxito de todo sistema orientado en su consecución. Para Rao, Solis y Raughunathan (1999) la alta dirección debe aceptar su responsabilidad para liderar la calidad y proporcionar un soporte activo y puntual para construir una conciencia sobre la misma, logrando, de este modo, un mayor nivel de rendimiento en las organizaciones (Ling, Simsek, Lubatkin \& Veiga, 2008).

El compromiso de la alta dirección con los sistemas de gestión de la calidad debe ir orientado hacia la consecución de los siguientes objetivos: 
- La integración de la gestión de la calidad en los valores, la visión, los planes y la estrategia de la empresa de forma clara, visible y consistente (Puffer \& McCarthy, 1996; Ahire \& O'Shaughnessy, 1998; Widodo, 2011).

- La responsabilidad en la creación del sistema organizacional que determinará cómo los productos y servicios son diseñados y producidos;

- El compromiso de los directivos en la mejora de los procesos, da prioridad a la filosofía de calidad sobre los costes, puesto que a largo plazo una calidad consistente y superior permitirá mejoras en costes y desempeño (Ahire, Golhar \& Waller, 1996; Kumar, De Grosbois, Choisne \& Kumar, 2008)

- La efectiva asignación de los recursos requeridos (Ahire \& O'Shaughnessy, 1998), en particular los recursos humanos, mediante la formación de los empleados en el uso de nuevos principios y herramientas de gestión de la calidad (Kaynak, 2003) y financieros (Ahire et al., 1996) para la correcta implantación de sistemas de gestión de la calidad.

A continuación, para la formación de una cultura orientada a la gestión de la calidad, la alta dirección debe impulsar la orientación al cliente como un compromiso por parte de todos los miembros de la organización. La orientación al cliente ha sido considerada como el principal objetivo de una cultura orientada la calidad (Samson \& Terziovski, 1999; Chiles \& Choi, 2000). La orientación al cliente implica un cambio de tendencia de las organizaciones tradicionales centradas en el coste y la eficiencia a organizaciones orientadas en la respuesta rápida a las demandas de los clientes con nuevas ideas y tecnologías, la producción de productos que satisfaga o exceda las expectativas de los clientes y la anticipación de lo que quieren y necesitan los clientes como requisito para el éxito organizacional a largo plazo (Ahire et al., 1996).

La orientación al cliente supone la determinación de la actuales y potenciales necesidades y expectativas de los consumidores (Samson \& Terziovski, 1999). A continuación, estas necesidades detectadas se convierten en el input para la gestión de la calidad, tratando de crear unos productos y servicios que, mediante el cumplimiento de unas determinadas especificaciones, cubran o excedan las necesidades anteriormente señaladas. En resumen, este principio implica la permanente búsqueda de la satisfacción del cliente a través de la continua adaptación a sus necesidades (Dean \& Bowen, 1994; Hill \& Wilkinson, 1995, Sun, 2011). Por eso, autores como Martinez-Lorente, Gallego-Rodriguez y Dale (1998) destacan la importancia que tiene para la organización que las necesidades de los clientes y su satisfacción estén siempre en la mente de todos los empleados, requisito imprescindible para su identificación.

Del mismo modo, una cultura organizativa que apuesta por la gestión de la calidad debe estar orientada hacia la creación de un entorno de trabajo que permita comprometer a los 
empleados en un proceso de cambio superando, de este modo, las complejidades operativas, las presiones del mercado y aquellos impedimentos que pudieran surgir (Kaynak, 2003; Sureshchandar, Rajemdr et al., 2003). Para autores como Dean y Evan (1994) la implantación de sistemas de gestión de la calidad implica la creación de un entorno de trabajo caracterizado por el trabajo en equipo, una mayor comunicación y cooperación entre los distintos departamentos y una mayor grado de responsabilidad de los empleados en la toma de decisiones para resolver los problemas y los cambios necesarios. Igualmente, Stetzer y Morgeson (1997) sugieren que las organizaciones que adoptan con éxito un sistema de gestión de la calidad tendrán un entorno de trabajo caracterizado por la cooperación, la confianza entre los directivos y empleados, ya que éstos deben sentir que la organización apoya sus esfuerzos orientados a la calidad. Dean \& Evans (1994) determinan que la implantación de sistemas de gestión de la calidad promueve la creación de un clima de trabajo caracterizado por la confianza, y la colaboración o cooperación entre los miembros de la organización y la alta dirección.

Finalmente, destacamos otros trabajos como Zeitz et al. (1997) que proponen que para la implantación con éxito de sistemas de gestión de la calidad se deben crear entornos de trabajo caracterizados, principalmente, por la comunicación entre la alta dirección y los empleados, la confianza -especialmente entre directivos y empleados-, y la cohesión social, a través de la calidad como soporte de las relaciones entre los trabajadores de la empresa. La gestión adecuada del entorno de trabajo por parte de las organizaciones repercute, entre otros aspectos, en mejoras de la productividad de los trabajadores (Cequea, Rodriguéz-Monroy \& Núñez-Bottini, 2011).

En síntesis, de acuerdo a estas argumentaciones, podemos plantear que las organizaciones que implantan o quieren implantar sistemas de gestión de la calidad tienen que promover una cultura organizativa orientada la creación de un entorno de trabajo basado en la confianza y percepción de un compromiso para desarrollar una comunicación abierta entre los miembros de una organización y sus superiores.

\section{Sistemas avanzados de gestión de los recursos ERP}

El uso de nuevas tecnologías de la información en la empresa se ha convertido en una necesidad estratégica para competir en la mayor parte de industrias, aunque el mero esfuerzo de poseer un determinado software no garantiza la mejora de la posición competitiva de la organización (Yusuf, Gunasekaran \& Abthorpe, 2004; Ray, Muhanna \& Barney, 2005). En este contexto competitivo, muchas empresas han optado por usar sistemas conocidos como ERP que guían la transmisión y proceso de toda la información necesaria para la sincronización de la toma de decisiones (Su \& Yang, 2010). 
Existe una amplia variedad de definiciones que recogen los distintos aspectos del ERP, como la propuesta por Mabert, Soni y Venkataramanan (2001) que lo define como un sistema completo diseñado para integrar y optimizar los diferentes procesos de negocio desarrollados por la empresa. Este sistema soporta las operaciones de negocio del día a día, ayudando al proceso de toma de decisiones (Hitt, Wu \& Zhou, 2002). Mientras unas están centradas en el concepto en sí, otras hacen referencia al ERP como sistema. En este sentido, Jacobs \& Bendoly (2003) determinan que una definición orientada al concepto de ERP hace referencia a un uso operativo y estratégico de esta tecnología, mientras que la orientación hacia el sistema hace referencia a la correcta implantación y manutención del ERP. En el presente artículo se opta por la orientación hacia el sistema.

De este modo, el concepto de ERP dista del sistema ERP en tanto que este último no lo considera como un simple conjunto de herramientas para gestionar procesos, sino como una infraestructura tecnológica diseñada para soportar las capacidades de todas las herramientas y procesos usadas en la empresa, de tal forma que aseguran los objetivos de flexibilidad de los procesos operativos ( $\mathrm{Ng}$ et al., 1999; Bendoly, 2003). Otros autores definen, a la hora de gestionar un sistema ERP que ya funciona de forma operativa y estable en la empresa, el concepto de competencia en ERP como una cartera de recursos directivos, técnicos, organizativos y de conocimiento experto (Stratman \& Roth, 2002).

\section{Beneficios de la implantación de un sistema ERP}

El impacto de las Tecnologías de la Información (TI) en el rendimiento de la empresa es una de las cuestiones más importantes tanto para investigadores como para directivos. Una de las principales características de un ERP es que permite compartir una gran cantidad de información, afectando a todos los procesos principales de una empresa (Kalling, 2003). De este modo, aunque no existe un consenso sobre el impacto positivo del sistema ERP sobre el rendimiento (Brynjolfsson \& Hitt, 2000; Hitt et al., 2002; Mabert, Soni \& Venkataramanan, 2003; Banker, Bardhan, Chang \& Lin, 2006), existe evidencia empírica del impacto favorable sobre la gestión de la cadena de suministro (Hsu, Tan, Kannan \& Leong, 2009; Su \& Yang, 2010) o la gestión de las relaciones con los clientes (Hendricks, Singhal \& Stratman, 2007).

Independientemente del efecto sobre el rendimiento de la empresa, existen diferentes autores que abordan el estudio de los beneficios del ERP, entre los que destacan Gattiker y Goodhue (2004) que los agrupan en cuatro categorías relacionadas con la mejora del flujo de información, la centralización de las principales actividades de gestión, la reducción de costes del sistema de información y el alcance de las mejores prácticas en la gestión de procesos. Yusuf et al. (2004) agrupan los principales beneficios del ERP en tres dimensiones: Automatización de los procesos de negocio, acceso en tiempo real a la información y mejora de la cadena de suministro mediante el uso del comercio electrónico. 
Entre las mejoras proporcionadas por un sistema ERP citamos a Holt (1999), el cual hace hincapié en la mejora de eficiencia derivada del incremento en la velocidad del flujo de la información. Otros autores señalan que reduce el tiempo que se tarda en atender la orden de un cliente (Cotteleer \& Bendoly, 2006), se determinan mejor los ciclos financieros (Mabert et al., 2003), se produce una integración más efectiva con clientes o proveedores (Stratman \& Roth, 2002), se favorece la visión compartida (Kearny, 2000) o se facilitan los procesos directivos de las empresas (Scott \& Vessey, 2000).

En el presente estudio se ha seleccionado la clasificación propuesta por Shang y Sheddon (2000), empleada en trabajos recientes como el de Su y Yang (2010), que distingue entre cinco grupos de beneficios genéricos: Beneficios relativos de la Infraestructura de TI (flexibilidad, usabilidad y eficiencia en coste), operativos (reducción de costes, mejora de la productividad, la calidad, la atención al cliente, etc.), directivos (relaciones con otros agentes, toma de decisiones y planificación), estratégicos (alianzas, innovaciones, mejoras en diferenciación y coste, etc.) y organizativos (aprendizaje, empowerment, nuevas pautas de comportamiento, etc.).

\section{Marco de investigación, modelo e hipótesis}

En la literatura encontramos diferentes trabajos teóricos y empíricos que analizan y verifican la relación entre la implantación de un sistema de Gestión Total de la Calidad y la mejora del desempeño en las organizaciones.

Un primer grupo de trabajos teóricos sugiere que la calidad puede conducir a la obtención de un mayor desempeño a través de dos enfoques diferentes (Garvin, 1988; Deming 1986; Reed, Lemak \& Montgomery, 1996). Desde el enfoque interno, la calidad produce una mejora de la fiabilidad y la conformidad de los productos y esto repercute en una disminución de los costes de fabricación y servicios. Desde un enfoque externo, la personalización de un producto para satisfacer las necesidades de los clientes puede incrementar su satisfacción, lo cual tiene efectos directos sobre la lealtad y retención de los clientes. Por consiguiente, incrementa las ventas futuras e incluso disminuye los costes mediante el logro de economías de escala. En ambos enfoques la calidad implica mejora en la gestión de los clientes y recursos de la empresa, lo que redunda en un incremento tanto en los beneficios operativos y como financieros de la organización.

Otros trabajos teóricos han analizado la relación entre el desarrollo de una cultura orientada a la gestión de la calidad y los beneficios que reporta a la organización a través de la teoría de recursos y capacidades (Powell, 1995; Savolainen, 2000). Una cultura de calidad orientada al compromiso de la alta dirección, el enfoque al cliente y la confianza de los miembros de la organización, permite el desarrollo de recursos valiosos caracterizados por ser específicos, 
producir relaciones sociales complejas y generar conocimiento tácito (Powell, 1995). Igualmente, permite el desarrollo de una serie de rutinas y comportamientos organizativos que hacen difícil que sus competidores puedan reproducir con éxito la misma estrategia (Savolainen, 2000). Finalmente, genera una serie de recursos intangibles responsables de la obtención de competencias distintivas, lo que contribuye a una mejora de la gestión de los recursos tangibles de la empresa y al incremento del desempeño organizativo.

En relación con los trabajos empíricos destacamos a White (1996) que analiza el efecto positivo de la implantación de la calidad en los beneficios operativos y financieros de la organización. Pero existen otros trabajos que analizan en que medida los elementos de una cultura orientada la calidad influyen en los distintos beneficios de la organización. Samson y Terzlovski (1999) analizan como la orientación al cliente o la confianza y el compromiso del personal de la organización influyen significativamente en el desempeño operativo de la organización. Powell (1995) estudia como el compromiso de la dirección o la adopción de una comunicación abierta influyen en los beneficios financieros de la organización; Hasan y Kerr (2003) muestran que las dimensiones compromiso de la alta dirección y enfoque cliente son las más importantes en términos de sus efectos sobre el desempeño organizacional. De la Maza-Aramburu, Vendrell-Herrero y Wilson (2012) analizan la relación entre la implantación de sistemas de gestión de la calidad y el desempeño innovador y, finalmente, Gustafsson y Nilsson (2003) indican que los resultados de negocio están influenciados por elementos como la gestión de los recursos humanos o la orientación al cliente.

Por otra parte, se comprueba que una cultura organizativa caracterizada por el compromiso de la alta dirección con los sistemas de gestión de la calidad aumenta la probabilidad de que los sistemas de gestión de la calidad sean implantados correctamente, y por tanto, la organización obtenga productos y servicios de calidad y mejores resultados. Así por ejemplo, Kaynak (2003) destaca la influencia positiva que ejerce el compromiso de la alta dirección sobre las prácticas de gestión de la calidad como la formación, las relaciones con los empleados, las relaciones con proveedores y el diseño del producto, lo cual mejora la implantación de los sistemas de gestión de calidad. Igualmente, Ugboro y Obeng (2000) o Howard y Foster (1999) demuestran que el compromiso de la alta dirección mejora los niveles de satisfacción de los trabajadores, lo cual lleva a una mejor prestación del servicio y satisfacción de los clientes y empleados. De este modo, la utilización de herramientas relacionadas con la gestión de la calidad en los procesos productivos mejora la asignación de los recursos por parte de las organizaciones, pudiendo afirmarse que:

Hipótesis Ha1 a Ha5: El compromiso de la alta dirección para promover una cultura basada en la calidad afecta positivamente a los beneficios operativos (Ha1), directivos (Ha2), estratégicos (Ha3), en cuestión de infraestructura de tecnologías de la información (Ha4) y organizacionales (Ha5) derivados de la implantación de un sistema de gestión de recursos ERP 
Toda esta argumentación permite afirmar que cuando la orientación organizativa es hacia la calidad es imprescindible un adecuado entendimiento de las necesidades de los clientes (Samson \& Terziovski, 1999). El uso de este conocimiento en el diseño de mejores productos y servicios, así como una orientación de la calidad hacia el cliente tiene un efecto directo sobre su satisfacción (Hill \& Wilkinson, 1995; Sun, 2011). Este proceso tiene a su vez un impacto positivo sobre la retención de cliente y el éxito de la organización a largo plazo derivado de un mejor tratamiento de las reclamaciones que se trasladan en futuras mejoras de los procesos de la empresa. Partiendo de esta argumentación, puede afirmarse que:

Hipótesis Hb1 a Hb5: El compromiso de control y mejora del rendimiento derivado de la gestión de clientes de la empresa afecta positivamente a los beneficios operativos (Hb1), directivos ( $\mathrm{Hb2}$ ), estratégicos ( $\mathrm{Hb} 3)$, en cuestión de infraestructura de tecnologías de la información (Hb4) y organizacionales (Hb5) derivados de la implantación de un sistema de gestión de recursos ERP

En la misma línea, el compromiso con la calidad en las organizaciones refuerza la confianza de los empleados ya que les permite tener certeza de que el proceso de regulación de la calidad de los procesos de la empresa es riguroso (Dean \& Evans, 1994). La contrastación de ideas con la alta dirección es frecuente, mostrándose mutua confianza entre unos y otros (Foster et al., 2002). Esta confianza se refleja en una mejora de los procesos, siendo factible afirmar que:

Hipótesis Hc1 a Hc5: La confianza mutua entre empleados y dirección en la gestión de una cultura orientada hacia la calidad derivada de la implantación de una comunicación abierta entre ambas partes afecta positivamente a los beneficios operativos (Hc1), directivos (Hc2), estratégicos (Hc3), en cuestión de infraestructura de tecnologías de la información (Hc4) y organizacionales (Hc5) derivados de la implantación de un sistema de gestión de recursos ERP

Planteadas las hipótesis de partida, el conjunto de relaciones se recoge en la figura 1 , procediéndose a continuación a su contrastación empírica.

\section{Diseño de la investigación y estudio empírico}

\section{Muestra}

Para comprobar las hipótesis se realiza un estudio empírico utilizando la base de datos SABI que contiene información sobre las principales empresas que operan en España. Se seleccionaron las empresas pertenecientes al sector manufacturero por ser un sector intensivo en el uso de la TI (Heim \& Peng, 2010). De esta base se obtuvieron un total de 1.208 empresas que mostraban una orientación hacia la calidad y, además, tenían implantado un sistema ERP. 


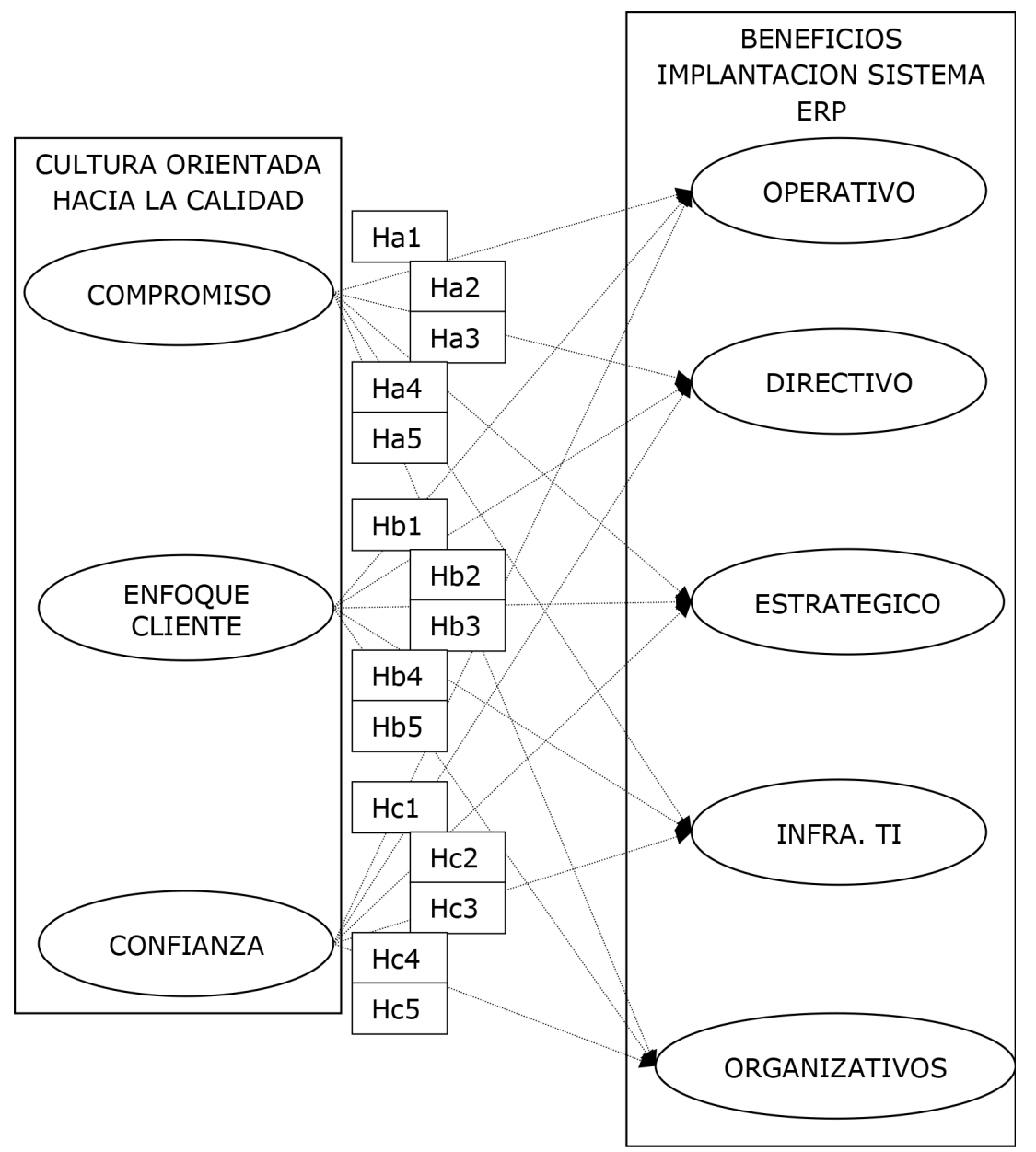

Figura 1. Modelo de relaciones entre las variables e hipótesis propuestas

El contacto con las empresas seleccionadas se realizó a través de una entrevista telefónica asistida por ordenador (CATI). El cuestionario iba dirigido al responsable del área de TI así como al director general de la empresa. La contestación del cuestionario se realizó durante los meses de Octubre y Noviembre de 2010. Se obtuvieron 205 encuestas válidas, siendo la tasa de respuesta del 26,67\%, tasa similar a la de otros estudios de dirección de operaciones (Bustinza, Molina \& Gutiérrez-Gutiérrez, 2010). La descripción completa de la muestra se incorpora en la tabla 1. Para garantizar la valía del encuestado, se le preguntó sobre su conocimiento sobre las áreas investigadas, su nivel de educación, antigüedad en el puesto de trabajo y antigüedad en la empresa. Del mismo modo, se realizaron diferentes pruebas T para determinar si existían diferencias muestrales en relación al tamaño, experiencia de las empresas o distribución sectorial que pudiesen matizar los resultados obtenidos, no encontrándose diferencias significativas entre los grupos seleccionados.

Para evaluar las vías de no respuesta se llevó a cabo el procedimiento de Armstrong y Overton (1977), que compara a través de two-tailed t-tests si existen diferencias en función de un conjunto de variables demográficas entre los que respondieron primero la encuesta y los que 
tardaron más en el tiempo. A un nivel $p<0.01$ no se observaron diferencias, por lo que las vías de no respuesta no aparecen como un problema significativo en el presente estudio.

\begin{tabular}{|c|c|}
\hline Fuente & SABI Sistema de Análisis de Balances Ibéricos \\
Área geográfica & España \\
Instrumento de medición & Cuestionario estructurado \\
Procedimiento & CATI Entrevistas telefónicas \\
Método de recolección & Muestreo simple sin reposición \\
Universo & Empresas con más de 20 empleados \\
Población & 1.208 empresas \\
Tamaño muestral & 205 empresas \\
Índice de respuesta & $26,67 \%$ \\
Error muestral & $6,1 \%$ \\
Edad & Sector \\
Periodo de recolección de datos & O-20 (34\%) / 20-40 $(44 \%) /+40(22 \%)$ \\
\hline
\end{tabular}

Tabla 1. Especificaciones técnicas de la muestra

\section{Medidas y justificación}

\section{Cultura Orientada hacia la calidad en la empresa}

Con el objetivo de medir este constructo se utiliza una escala Likert de 7 puntos (desde 1 = Totalmente en desacuerdo a $7=$ Totalmente de acuerdo) de acuerdo a la escala de la cultura orientada hacia la calidad en la empresa establecida por Jayaram, Ahire y Dreyfus (2010). Estos autores planteaban tres dimensiones para diseñar adecuadamente un sistema que cumpliera estas premisas, por lo que, en este sentido, puede entenderse que es igualmente útil para el presente estudio. Por lo tanto, se plantean dimensiones de medición del compromiso, el enfoque hacia el cliente y la confianza como elementos esenciales de la cultura hacia la calidad.

El análisis de componentes principales muestra la existencia de estas tres dimensiones, arrojando valores para el Alpha de Cronbach de $a_{1}=0,895$ para la medición del compromiso en el sistema, $a_{2}=0,833$ para el enfoque hacia el clientes, y $a_{3}=0,870$ para la confianza, dotando a estas dimensiones con la correspondiente consistencia interna para su utilización en el modelo planteado.

\section{Beneficios de la implantación de un sistema ERP}

En este caso se plantea una escala Likert de 7 puntos (desde $1=$ Totalmente en desacuerdo a 7 = Totalmente de acuerdo) recogiendo los cinco beneficios esenciales derivados de un sistema ERP (Shang \& Seddon, 2000; Su \& Yang, 2010). Estos beneficios son de tipo operacional, directivo, estratégico, en infraestructuras de TI y organizacional, y Su y Yang (2010) los establecen para estudiar cómo afecta el funcionamiento de un sistema ERP y los beneficios que genera. 
La consistencia interna de la escala y su fiabilidad son estudiadas mediante un análisis factorial exploratorio. El análisis de componentes principales constata que existen las cinco dimensiones previstas para este constructo, mientras que los valores del Alpha de Cronbach son de $\mathrm{a}_{1}=0,865$ para los beneficios operacionales de un sistema ERP, $\mathrm{a}_{2}=0,905$ para los directivos, $a_{3}=0,912$ para los estratégicos, $a_{4}=0,886$ para los derivados de infraestructuras en TI $y$, finalmente, $a_{5}=0,937$ para los organizacionales. Estos valores determinan la consistencia interna de la escala validándola para su utilización.

La traducción del conjunto de escalas utilizadas en el presente estudio se recoge en el Apéndice 1.

\section{Metodología y resultados obtenidos}

El modelo de relaciones propuesto se analiza a través del planteamiento de un sistema de ecuaciones estructurales SEM (Structural Equations Modelling), tratado con el paquete estadístico EQS 6.2. Los resultados de este análisis se aprecian en la tabla 2 donde se recogen los estadísticos descriptivos, las fiabilidades y varianzas extraídas de las distintas escalas utilizadas en este estudio. A la vista de los valores obtenidos se puede asegurar la validez convergente de las escalas y la idoneidad de su utilización.

Los resultados del análisis estructural se presentan en la tabla 3. En primer lugar se estudia la bondad de ajuste del modelo a partir de los indicadores y las recomendaciones respecto a su valor que aparecen en Hair, Anderson, Tatham y Black (2004). Para ello, se consideran tres tipos de indicadores: medidas de la bondad de ajuste absoluto, incremental y de parsimonia. Respecto al primer grupo de indicadores, hemos seleccionado el índice de bondad de ajuste (GFI), el error de aproximación cuadrático medio (RMSEA) y el error cuadrático medio (RMR). Por otra parte, es necesario también comprobar que el modelo presenta un buen ajuste incremental. El último aspecto de estudio es el ajuste de la parsimonia del modelo, mostrándose todos los valores obtenidos en la tabla 3.

Los resultados de los análisis están en línea con la hipótesis general del estudio, en definitiva, que un sistema diseñado con una premisa de orientación hacia la calidad tiene efectos positivos sobre un sistema de gestión de recursos ERP. No obstante, es preciso matizar los resultados obtenidos analizando el valor de los respectivos parámetros de cada hipótesis realizando la correspondiente discusión de los resultados. 


\begin{tabular}{|c|c|c|c|c|c|}
\hline \multicolumn{6}{|c|}{ Indicadores de la variable "cultura hacia la calidad" } \\
\hline ITEM & Carga Factorial (t) & Fiabilidad $\left(\mathbf{R}^{2}\right)$ & ITEM & Carga Factorial (t) & Fiabilidad $\left(\mathbf{R}^{2}\right)$ \\
\hline CAL11 & $0,875(8,714)$ & 0,765 & CAL24 & $0,708(7,815)$ & 0.501 \\
\hline CAL12 & $0,864(7,766)$ & 0,746 & CAL25 & $0,732(7,274)$ & 0.535 \\
\hline CAL13 & $0,727(7,919)$ & 0,528 & CAL31 & $0,710(7,993)$ & 0.504 \\
\hline CAL14 & $0,741(7,188)$ & 0,549 & CAL32 & $0,853(8,430)$ & 0.727 \\
\hline CAL15 & $0,778(8,082)$ & 0,605 & CAL33 & $0,848(8,213)$ & 0.719 \\
\hline CAL21 & $0,808(7,432)$ & 0,652 & CAL34 & $0,817(7,987)$ & 0.667 \\
\hline CAL22 & $0,797(7,289)$ & 0,635 & \multicolumn{3}{|c|}{ Varianza extraída 0.514} \\
\hline CAL23 & $0,737(8,269)$ & 0,543 & \multicolumn{3}{|c|}{ Fiabilidad compuesta 0.936} \\
\hline \multicolumn{6}{|c|}{ Indicadores de la variable "beneficios de un sistema ERP" } \\
\hline ITEM & Carga Factorial (t) & Fiabilidad $\left(\mathbf{R}^{2}\right)$ & ITEM & Carga Factorial (t) & Fiabilidad $\left(\mathbf{R}^{2}\right)$ \\
\hline BERP11 & $0,761(10,269)$ & 0,579 & BERP35 & $0,694(11,351)$ & 0,481 \\
\hline BERP12 & $0,644(10,253)$ & 0,415 & BERP36 & $0,815(10,737)$ & 0,664 \\
\hline BERP13 & $0,670(10,815)$ & 0,449 & BERP41 & $0,829(9,427)$ & 0,687 \\
\hline BERP14 & $0,776(11,563)$ & 0,602 & BERP42 & $0,772(9,767)$ & 0,595 \\
\hline BERP15 & $0,821(11,944)$ & 0,674 & BERP43 & $0,834(9,669)$ & 0,695 \\
\hline BERP16 & $0,832(12,849)$ & 0,692 & BERP44 & $0,684(9,258)$ & 0,468 \\
\hline BERP21 & $0,663(10,896)$ & 0,439 & BERP45 & $0,633(9,015)$ & 0,401 \\
\hline BERP22 & $0,758(10,527)$ & 0,574 & BERP46 & $0,693(9,806)$ & 0,480 \\
\hline BERP23 & $0,830(11,711)$ & 0,688 & BERP51 & $0,734(10,979)$ & 0,538 \\
\hline BERP24 & $0,812(12,323)$ & 0,659 & BERP52 & $0,865(11,609)$ & 0,748 \\
\hline BERP25 & $0,789(12,025)$ & 0,622 & BERP53 & $0,845(10,181)$ & 0,786 \\
\hline BERP26 & $0,805(12,269)$ & 0,648 & BERP54 & $0,826(11,093)$ & 0,714 \\
\hline BERP31 & $0,867(11,353)$ & 0,751 & BERP54 & $0,865(11,532)$ & 0,682 \\
\hline BERP32 & $0,811(11,364)$ & 0,657 & BERP56 & $0,694(12,101)$ & 0,748 \\
\hline BERP33 & $0,866(10,550)$ & 0,749 & & Varianza extraída & 08 \\
\hline BERP34 & $0,742(11,284)$ & 0,551 & & Fiabilidad compuest & 968 \\
\hline
\end{tabular}

Tabla 2. Carga factorial y análisis de fiabilidad de las escalas 


\begin{tabular}{|c|c|c|c|c|}
\hline \multirow{4}{*}{ Tipo de ajuste } & Indicador & Nomenclatura & Rango aceptación & Valores \\
\hline \multirow{4}{*}{ Absoluto } & Chi-Square Likelihood & CMIN & Ofrece test significación & $\begin{array}{c}860,323 \\
(p=0,326)\end{array}$ \\
\cline { 2 - 5 } & Goodness-of-Fit Index & GFI & $>0,900$ & 0,973 \\
\cline { 2 - 5 } & Root Mean Square Error & RMSEA & $0,050-0,080$ & 0,054 \\
\cline { 2 - 5 } & Root Mean Residual & RMR & $<0,050$ & 0,039 \\
\hline \multirow{3}{*}{ Incremental } & Compared Fit Index & CFI & $>0,900$ & 0,987 \\
\cline { 2 - 5 } & Normed Fit Index & NFI & $>0,900$ & 0,965 \\
\cline { 2 - 5 } & Adjusted Goodness Fit & NGFI & $>0,900$ & 0,984 \\
\hline \multirow{2}{*}{ Parsimonia } & Normed Chi-square & CMINDF & $>0,900$ & 0,964 \\
\hline
\end{tabular}

Tabla 3. Indicadores de la bondad de ajuste absoluto, incremental y de parsimonia del modelo propuesto

\section{Discusión}

El efecto del compromiso en un sistema orientado hacia la calidad en los beneficios de un sistema de gestión de recursos ERP. Análisis de los beneficios de tipo operativo, directivo y estratégico

Como se puede apreciar en la tabla 4, los valores de los parámetros de la relación entre el compromiso en la orientación hacia la calidad y los BERP son positivos y significativos en el caso del impacto en los de tipo operativo $\left(\beta_{1}=0,343 ; p<0,01\right)$, directivo $\left(\beta_{2}=0,264 ; p<0,01\right)$, estratégico $\left(\beta_{3}=0,512 ; p<0,01\right)$, en infraestructuras en TI $\left(\beta_{4}=0,541 ; p<0,01\right)$, y organizativos $\left(\beta_{5}=0,583 ; p<0,01\right)$, lo que permite aceptar las hipótesis Ha1 a Ha5.

Los sistemas ERP proporcionan beneficios operativos de distinta índole. Por un lado, aquellos resultantes de la automatización de procesos multifuncionales (Yusuf et al., 2004). Por otro, los beneficios derivados de la utilización de datos que permiten mejorar la planificación y dirección de la producción, de la carga de trabajo, de los inventarios y de los recursos físicos (AranaSolares et al., 2012). Y, finalmente, los beneficios que surgen de un seguimiento y control más exhaustivo del rendimiento financiero de los diferentes productos, clientes, y áreas de negocio y geográficas de la empresa (Hitt et al., 2002; Banker et al., 2006). A la vista de los resultados obtenidos, puede afirmarse que el compromiso de los directivos con una cultura hacia la calidad, la gestión de los clientes desde una óptica de compromiso con la calidad, y la confianza por parte de los trabajadores en que la empresa está comprometida con la calidad tienen un efecto directo y significativo sobre todo el conjunto de beneficios estratégicos derivados de la utilización de un sistema de gestión de recursos ERP. 


\begin{tabular}{|c|c|c|c|}
\hline \multicolumn{2}{|c|}{ Modelo estructural } & Coeficiente & Acceptacion/ rechazo \\
\hline \multirow{5}{*}{ Compromiso } & Beneficios operativos & $0,343(\mathrm{t}=3,933) * * *$ & Ha1: aceptada \\
\hline & Beneficios directivos & $0,364(t=3,418)^{* * *}$ & Ha2: aceptada \\
\hline & Beneficios estratégicos & $0,312(t=3,897)^{* * *}$ & Ha3: aceptada \\
\hline & Beneficios infraes. TI & $0,341(t=3,521)^{* * *}$ & Ha4: aceptada \\
\hline & Beneficios organizativos & $0,383(\mathrm{t}=3,738)^{* * *}$ & Ha5: aceptada \\
\hline \multirow{5}{*}{ Enfoque en el cliente } & Beneficios operativos & $0,514(\mathrm{t}=4,163)^{* * *}$ & Hb1: aceptada \\
\hline & Beneficios directivos & $0,513(t=4,478)^{* * *}$ & Hb2: aceptada \\
\hline & Beneficios estratégicos & $0,459(t=3,670)^{* * *}$ & Hb3: aceptada \\
\hline & Beneficios infraes. TI & $0,183(t<1,354)$ & Hb4: rechazada \\
\hline & Beneficios organizativos & $-0,043(t<0,907)$ & Hb5: rechazada \\
\hline \multirow{5}{*}{ Confianza } & Beneficios operativos & $0,577(\mathrm{t}=4,231) * * *$ & Hc1: aceptada \\
\hline & Beneficios directivos & $0,636(\mathrm{t}=4,879)^{* * *}$ & Hc2: aceptada \\
\hline & Beneficios estratégicos & $0,539(\mathrm{t}=3,765)^{* * *}$ & Hc3: aceptada \\
\hline & Beneficios infraes. TI & $0,602(\mathrm{t}=3,672) * * *$ & Hc4: aceptada \\
\hline & Beneficios organizativos & $0,638(\mathrm{t}=4,009)^{* * *}$ & Hc5: aceptada \\
\hline \multicolumn{4}{|c|}{ Nivel de significación: $* * * p<0.001$} \\
\hline
\end{tabular}

Tabla 4. Aceptación/rechazo de hipótesis

En cuanto a los beneficios de tipo directivo, hacen referencia a los efectos que estos sistemas tienen en el largo plazo. Se trataría de los beneficios derivados de la mejora en los procesos de negocio del día a día, de la mayor capacidad de respuesta ante las necesidades de los clientes mejorando su satisfacción, de la mayor capacidad para entregar en los plazos requeridos, y de la mejora de los procesos de toma de decisión (Scott \& Vessey, 2000; Su \& Yang, 2010). En este sentido, el compromiso de la empresa, la orientación hacia el cliente y la confianza generada por la cultura orientada hacia la calidad son determinantes del conjunto de beneficios de tipo directivo.

En lo referente a los beneficios de índole estratégico, surgen por la habilidad del sistema para apoyar el crecimiento de negocio, reduciendo el coste de mantenimiento del inmovilizado de la empresa, recogiendo, del mismo modo, los beneficios derivados del aprendizaje de negocio y de la mayor satisfacción del empleado con el trabajo realizado (Jacobs \& Bendoly, 2003). En este punto, la orientación organizativa hacia la calidad del sistema demuestra afectar positiva y significativamente al conjunto de beneficios estratégicos que subyacen a la utilización de sistemas avanzados de gestión de recursos como el ERP. 
El efecto del enfoque en clientes y la confianza de un sistema orientado hacia la calidad en los beneficios de un sistema de gestión de recursos ERP. Análisis de los beneficios en infraestructuras en TI y organizativos

En cuanto a los valores de los parámetros de la relación entre el enfoque hacia el cliente y los BERP, tabla 4, sólo son positivos y significativos en el caso del impacto en los de tipo operativo $\left(\beta_{6}=0,514 ; p<0,01\right)$, directivo $\left(\beta_{7}=0,513 ; p<0,01\right)$, y estratégico $\left(\beta_{8}=0,459 ; p<0,01\right)$, lo que permite aceptar las hipótesis $\mathrm{Hb} 1 \mathrm{a} \mathrm{Hb3}$. Sin embargo, el parámetro de la relación entre este enfoque $y$ los beneficios en infraestructuras en TI es positivo pero no significativo $\left(\beta_{9}=0,183 ; t<1,354\right)$, mientras que el parámetro que lo relaciona con los BERP organizativos es negativo y no significativo $\left(\beta_{10}=-0,061 ; p<0,01\right)$, lo que implica el rechazo de las hipótesis $\mathrm{Hb} 4$ y $\mathrm{Hb} 5$.

Por último, y como igualmente se recoge en la tabla 4, el valor de los parámetros de la relación entre la confianza en un sistema orientado hacia la calidad y los BERP de tipo operativo $\left(\beta_{11}=0,577 ; p<0,01\right)$, directivo $\left(\beta_{12}=0,636 ; p<0,01\right)$, estratégico $\left(\beta_{13}=0,539 ; p<0,01\right)$, en infraestructuras en TI $\left(\beta_{14}=0,602 ; p<0,01\right)$, y organizativos $\left(\beta_{1}=0,638 ; p<0,01\right)$ son positivos y significativos, aceptándose las hipótesis Hc1 a Hc5.

En cuanto a la discusión de los resultados, señalar que los beneficios derivados de la infraestructura de tecnologías de la información hacen referencia a la obtención de una mayor flexibilidad de negocio, y al aumento de la capacidad del sistema ( $\mathrm{Ng}$ et al., 1999). En este aspecto, hay que destacar que el compromiso de la alta dirección con la aplicación de premisas basadas en la gestión de la calidad y la confianza de los empleados en que estas premisas se cumplen rigurosamente afectan a los beneficios de la utilización de sistemas ERP, pero no se encuentra significativa la influencia del enfoque en el cliente en este conjunto de beneficios. La justificación se encuentra en el hecho de que el enfoque en la atención a las reclamaciones del cliente y la búsqueda de su satisfacción no suponen, en ningún caso, aumentos de la capacidad del sistema ni de la flexibilidad de negocio ya que para atender sus reclamaciones hay que destinar recursos y tiempo.

Finalmente, los beneficios organizativos son aquellos relativos al apoyo a los procesos de cambio soportados por el sistema, al refuerzo del sentimiento de pertenencia a la organización por parte de los empleados, y a la construcción de una visión compartida general a todos los miembros de la organización (Schein, 2004; Suppiah \& Sandhu, 2011). En este apartado, se comprueba que hay una relación directa y significativa entre el compromiso de la alta dirección y la confianza del personal en la aplicación de una cultura efectiva orientada hacia la calidad en los procesos, sin encontrarse relación -como en el caso de los beneficios de la infraestructura en tecnologías de la información- entre el enfoque en el cliente y los citados beneficios organizativos. La justificación se encuentra en el hecho de que en el enfoque al cliente suele 
señalarse con más precisión un responsable de las reclamaciones de los clientes, siendo el personal de atención al cliente el que más sufre de reprobaciones que, en muchos casos, pueden afectar al ámbito personal del empleado. En último término, estas controversias entre clientes y empleados pueden significar para el empleado involucrado una desvinculación con la empresa al no sentirse debidamente respaldado por sus superiores frente al cliente (de hecho aunque no se encuentra significación en el parámetro, éste era negativo lo que puede indicar tendencia en ese sentido, es decir, efecto negativo de la orientación hacia el cliente en los beneficios organizativos de un sistema ERP).

\section{Conclusiones, limitaciones y futuras líneas de investigación}

El apoyo de la alta dirección hacia una cultura basada en el compromiso con la calidad en las organizaciones supone un elemento esencial de la estrategia competitiva de las mismas (Flynn, Schroeder \& Sakakibara, 1995). Este compromiso tiene un efecto directo en la gestión de los procesos de la empresa, por lo que constituye un determinante de los beneficios que pudiera generar cualquier sistema de gestión de recursos aplicados a la dirección de dichos procesos (Dean \& Evans, 1994).

El análisis de los resultados permite corroborar que los sistemas enfocados en la satisfacción del cliente, como el que es objeto de estudio en el presente trabajo, afectan a la relación entre las organizaciones y su cartera de clientes (Ahire et al., 1996), redundando en que la percepción de los clientes respecto a los productos y servicios ofertados mejore (Kumar, De Grosbois, Choisne \& Kumar, 2008). Los sistemas de gestión de recursos ERP facilitan el intercambio de información entre clientes y organizaciones, lo que supone que bajo una orientación hacia la calidad en los procesos, el nivel de compromiso con la satisfacción del cliente aumenta.

En cuanto al análisis de las relaciones con los trabajadores de la empresa, el compromiso de la alta dirección en este sentido refuerza el nivel de comunicación entre los miembros de la organización (Zeitz et al., 1997; Cequea et al., 2011). Las premisas de la Gestión de la calidad suponen una confianza en la correcta gestión de los recursos y procesos de la empresa en los que la fuerza de trabajo representa uno de los recursos más valiosos.

Los resultados obtenidos refuerzan la necesidad por parte de las organizaciones de concentrar sus esfuerzos en una cultura hacia la calidad y el enfoque en la satisfacción del cliente, generando un compromiso por parte de los integrantes de la organización y un refuerzo en la confianza en que los procesos desarrollados en el seno de la misma se realizan bajo estas directrices (Stetzer \& Morgeson, 1997). La implantación de sistemas de gestión de recursos como el ERP garantizan una mejor gestión de los procesos señalados, reforzando el flujo de información que facilita que dichos procesos se realicen de forma más eficiente, lo que reporta 
en beneficios para las organizaciones de distinta índole (Stadtler \& Kilger, 2008). En definitiva, el compromiso de la alta dirección y de los trabajadores de la empresa en el cumplimiento de estas premisas en las que se fundamenta la Gestión de la calidad genera una cultura organizativa eficaz a la hora de gestionar los recursos de la empresa, lo que repercute en que sistemas como el ERP funcionen de manera más eficiente y se incremente el desempeño de los recursos a disposición de las organizaciones.

En relación con las limitaciones del presente estudio, se trata de un análisis estático que, aunque encuentra relaciones significativas entre las variables, puede dejar de lado otros factores importantes que desempeñen un papel importante en el contexto de los beneficios de este tipo de sistemas ERP.

Otras limitaciones, que pueden convertirse en futuras líneas de investigación, surgen al analizar la orientación hacia el cliente por parte de las organizaciones ya que limita la capacidad del sistema en el sentido de que es necesario destinar más recursos a la gestión de las reclamaciones de los clientes. Futuras investigaciones podrían esclarecer el papel de los recursos comprometidos en este sentido, y como este compromiso afecta a la asignación de recursos para otras partidas.

Del mismo modo, la búsqueda de responsabilidades cuando aparecen problemas en la gestión de las reclamaciones de los clientes puede suponer una dilución de responsabilidad y una falta de asunción de las mismas. Este aspecto debería ser estudiado con más detenimiento en el sentido de que es fuente de inestabilidad en las relaciones entre los trabajadores de la empresa. Aunque estas limitaciones están presentes, las conclusiones derivadas del presente estudio están en línea con la realidad que se observa en las organizaciones, permitiendo esclarecer determinados aspectos que una cultura organizativa orientada hacia la calidad tiene respecto a la gestión de los recursos a su disposición, y respecto a las relaciones entre la alta dirección y los empleados de dichas organizaciones.

\section{Referencias}

ADEBANJO, D.; KEHOE, D. (1998). An evaluation of quality culture problems in UK companies. International Journal of Quality Science, 3(3): 275-286. http://dx.doi.org/10.1108/13598539810370486

AHIRE, S.L.; GOLHAR, D.; WALLER, M. (1996). Development and validation of TQM implementation constructs. Decision Sciences, 27(1): 23-56. http://dx.doi.org/10.1111/j.15405915.1996.tb00842.x

AHIRE, S.L.; O'SAUCHNESSY, K.C. (1998). The role of top management commitment in quality management: An empirical analysis of the auto parts industry. International Journal of Quality and Reliability Management, 3(1): 5-37. 
ARANA-SOLARES, I.A; ALFALLA-LUQUE, R.; MACHUCA, J.A.D. (2012). Análisis de las variables que proporcionan una competitividad sostenible de la cadena de suministro. Intangible Capital, 8(1): 92-122.

ARMSTRONG, J.S.; OVERTON, T.S. (1977). Estimating non-response bias in mail surveys. Journal of Marketing Research, 14(3): 396-402. http://dx.doi.org/10.2307/3150783

BANKER, R.D.; BARDHAN, I.R.; CHANG, H.; LIN, S. (2006). Plant information systems, manufacturing capabilities, and plant performance. MIS Quarterly, 30(2): 315-337.

BENDOLY, E. (2003). Enterprise Resource Planning: Development and directions for operations management research. European Journal of Operational Research, 146(2): 233-240. http://dx.doi.org/10.1016/S0377-2217(02)00546-5

BRYNJOLFSSON, E.; \& HITT, L.M. (2000). Beyond computation: Information technology, organizational transformation and business performance. The Journal of Economic Perspectives, 14(4): 23-48. http://dx.doi.org/10.1257/jep.14.4.23

BUSTINZA, O.F.; MOLINA, L.M.; GUTIERREZ-GUTIERREZ, L. (2010). Outsourcing as seen from the perspective of Knowledge Management. Journal of Supply Chain Management, 46(3): 23-39. http://dx.doi.org/10.1111/j.1745-493X.2010.03196.x

CEQUEA, M.M.; RODRÍGUEZ MONROY, C.; NÚÑEZ BOTTINI, N.B. (2011). Intangible Capital, 7(2): 549-584.

CHILES, T.H.; CHOI, T.Y. (2000). Theorizing TQM: An Austrian and evolutionary economics interpretation. Journal of Management Studies, 37(2): 185-212. http://dx.doi.org/10.1111/1467-6486.00177

CHUNG, Y.C.; HSU, Y.W.; TSAI, C.H. (2010). Research on the correlation between implementation strategies of TQM, organizational culture, TQM activities and operational performance in high-tech firms. Information Technology Journal, 9(8): 1696-1705. http://dx.doi.org/10.3923/itj.2010.1696.1705

COTTELEER, M.J.; BENDOLY, E. (2006). Order lead-time improvement following enterprise information technology implementation: An empirical study. MIS Quarterly, 30(3): 643-660.

DE LA MAZA-ARAMBURU, X.; VENDRELL-HERRERO, F.; WILSON, J.R. (2012). Where is the value of cluster associations for firms? Intangible Capital, 8(2): 472-496. http://dx.doi.org/10.3926/ic.346

DEAN, J.W.; BOWEN, D.E. (1994). Management theory and total quality: Improving research and practice through theory development. Academy of Management Review, Vol. 19(3): 392-418.

DEAN, J.W.; EVANS, J.R. (1994). Total Quality Management, organization and strategy. StPaul: Wet Publishing. 
DEMING, W.E. (1986). Out of the crisis. Cambridge, MA: MIT Press.

DOUGLAS, T.J.; FREDENDALL, L.D. (2004). Evaluating the Deming management model and competitive advantage: The role of structural control and exploration. Academy of Management Journal, 44(1): 158-169. http://dx.doi.org/10.2307/3069343

FLYNN, B.B.; SCHROEDER, R.G.; SAKAKIBARA, S. (1995). The impact of quality management practices on performance and competitive advantage. Decision Sciences, 26(5): 659-692. http://dx.doi.org/10.1111/j.1540-5915.1995.tb01445.x

FOSTER, S.T.; HORWARD, L.W.; SHANNON, P. (2002). The role of quality tools in improving satisfaction with government. Quality Management Journal, 9(3): 20-31.

GARVIN, D.A. (1988). Managing quality: The strategic and competitive edge. New York: The Free Press.

GATTIKER, T.F.; GOODHUE, D.L. (2004). Understanding the local-level costs and benefits of ERP through organizational information processing theory. Information \& Management, 41(4): 431-443. http://dx.doi.org/10.1016/S0378-7206(03)00082-X

GUSTAFSSON, A.; NILSSON, L. (2003). The role of quality practices in service organizations. International Journal of Service Industry Management, 14(2): 232-244. http://dx.doi.org/10.1108/09564230310474183

HACKMAN, J.R.; WAGEMAN, R. (1995). Total Quality Management: Empirical, conceptual, and practical issues. Administrative Science Quarterly, 40(2): 309-342. http://dx.doi.org/10.2307/2393640

HAIR, J.F.; ANDERSON, R.E.; TATHAM, R.L.; BLACK, W.C. (2004). Análisis multivariante. Madrid: Prentice Hall.

HASAN, M.; KERR, R.M. (2003). The relationship between TQM practices and organizational performance in service organization. The TQM Magazine, 15(4): 286-291. http://dx.doi.org/10.1108/09544780310486191

HEIM, G.R.; PENG, D.X.J. (2010). The impact of information technology use on plant structure, practices, and performance: An exploratory study. Journal of Operations Management, 28(2): 144-162. http://dx.doi.org/10.1016/j.jom.2009.09.005

HENDRICKS, K.B.; SINGHAL, V.R.; STRATMAN, J.K. (2007). The impact of enterprise systems on corporate performance: A study of ERP, SCM, and CRM system implementations. Journal of Operations Management, 25(1): 65-82. http://dx.doi.org/10.1016/j.jom.2006.02.002

HILL, S.; WILKINSON, A. (1995). In search of TQM. Employee Relations, 17(3): 8-25. http://dx.doi.org/10.1108/01425459510147002 
HITT, L.M.; WU, D.J.; ZHOU, X. (2002). Investment in enterprise resource planning: Business impact and productivity measures. Journal of Management Information Systems, 19(1): 71-98.

HOLT, L. (1999). Competition heats up in ERP. Info World, 21(6): 65-75.

HOWARD, L.W.; FOSTER, S.T. (1999). The influence of human resource practices on empowerment and employee perceptions of management commitment to quality. Journal of Quality Management, 4(1): 5-22. http://dx.doi.org/10.1016/S1084-8568(99)80093-5

HSU, C.C.; TAN, K.C.; KANNAN, V.R.; LEONG, G.K. (2009). Supply chain management practices as a mediator of the relationship between operations capability and firm performance. International Journal of Production Research, 47(3): 835-855. http://dx.doi.org/10.1080/00207540701452142

JACOBS, F.R.; BENDOLY, E. (2003). Enterprise Resource Planning: Developments and directions for operations management research. European Journal of Operational Research, 146(2): 233-240. http://dx.doi.org/10.1016/S0377-2217(02)00546-5

JAYARAM, J.; AHIRE, S.L.; DREYFUS, P. (2010). Contingency relationships of firm size, TQM duration, unionization, and industry context on TQM implementation. A focus on total effects. Journal of Operations Management, 28(4): 345-356. http://dx.doi.org/10.1016/j.jom.2009.11.009

JONES, M.C.; CLINE, M.; RYAN, S. (2006). Exploring knowledge sharing in ERP implementation: An organizational culture framework. Decision Support Systems, 41(2): 411-434. http://dx.doi.org/10.1016/j.dss.2004.06.017

KALLING, T. (2003). ERP systems and the strategic management processes that lead to competitive advantage. Information Resources Management Journal, 16(4): 46-67. http://dx.doi.org/10.4018/irmj.2003100104

KAYNAK, H. (2003). The relationship between total quality management practices and their effects on firm performance. Journal of Operations Management, 7(1): 33-37.

KEARNY, A.T. (2000). Strategic information technology and the CEO agenda. Monograph. Chicago: A.T. Kearny Company.

KUMAR, V.; DE GROSBOIS, D.; CHOISNE, F.; KUMAR, U. (2008). Performance measurement by TQM adopters. TQM Journal, 20(3): 209-222. http://dx.doi.org/10.1108/17542730810867236

LEE, P.K.C.; CHENG, T.C.E.; YEUNG, A.C.L.; LAI, K.H. (2011). An empirical study of transformational leadership, team performance and service quality in retail banks. Omega, 39(6): 690-700. http://dx.doi.org/10.1016/j.omega.2011.02.001 
LING, Y.; SIMSEK, Z.; LUBATKIN, M.H.; VEIGA, J.F. (2008). The impact of transformational CEO's on the performance of small- to medium-sized firms: Does organizational context matter? Journal of Applied Psychology, 93(4): 923-934. http://dx.doi.org/10.1037/00219010.93.4.923

MABERT, V.A.; SONI, A.; VENKATARAMANAN, M.A. (2001). Enterprise Resource Planning: Measuring value. Production and Inventory Management Journal, 42(3/4): 46-51.

MABERT, V.A.; SONI, A.; VENKATARAMANAN, M.A. (2003). The impact of organization size on enterprise resource planning (ERP) implementations in the US manufacturing sector. Omega, 31(3): 235-246. http://dx.doi.org/10.1016/S0305-0483(03)00022-7

MARTINEZ-LORENTE， A.R.; GALLEGO-RODRÍGUEZ, A.; DALE， B.G. (1998). Total Quality Management and company characteristics: An examination. Quality Management Journal, 5(4): 59-71.

NG, J.K.C.; IP, W.H.; LEE, T.C. (1999). A paradigm for ERP and BPR integration. International Journal of Production Research, 37(9): http://dx.doi.org/10.1080/002075499190923

POWELL, T.C. (1995). Total Quality Management as competitive advantage: A review and empirical study. Strategic Management Journal, 16(1): 15-37. http://dx.doi.org/10.1002/smj.4250160105

PUFFER, S.M.; MCCARTHYM, D.J. (1996). A framework for leadership in a TQM context. Journal of Quality Management, 1(1): 109-130. http://dx.doi.org/10.1016/S1084-8568(96)90008-5

RAO, S.S.; SOLIS, L.E.; RAUGHUNATHAN, T.S. (1999). A framework for international quality management research: Development and validation of a measurement instrument. Total Quality Management, 10(7): 1047-1075. http://dx.doi.org/10.1080/0954412997226

RAY, G.; MUHANNA, W.A.; BARNEY, J.B. (2005). Information technology and the performance of the customer service process: A resource-based analysis. MIS Quarterly, 29(4): 625-652.

REED, R.; LEMAK, D.J.; MONTGOMERY, J.C. (1996). Beyond process: TQM content and firm performance. Academy of Management Review, 21(1): 173-202.

RUIZ-GUERRA, I.; MARTÍN LÓPEZ, V.M.; MOLINA MORENO, V. (2012). Los intangibles del Aceite de Oliva como ventaja competitiva. Intangible Capital, 8(1): 150-180.

SAMSON, D.; TERZIOVSKI, M. (1999). The relationship between total quality management practices and operational performance. Journal of Operations Management, 20(4): 810-829.

SAVOLAINEN, T. (2000). How organizations promote and avoid learning: Development of positive and negative learning cycles. Journal of Workplace Learning, 12(5): 195-204. http://dx.doi.org/10.1108/13665620010336198 
SCHEIN, E.H. (2004). Organisational culture and leadership. San Francisco, CA: Jossey-Bass.

SCHNIEDERJANS, M.J.; KIM, G.C. (2003). Implementing Enterprise Resource Planning systems with Total Quality control and business process reengineering: Survey results. International Journal of Operations \& Production Management, 23(4): 418-429. http://dx.doi.org/10.1108/01443570310467339

SCOTT, J.E.; VESSEY, I. (2000). Implementing Enterprise Resource Planning system: The role of learning from failure. Information System Frontiers, 2(2): 213-232. http://dx.doi.org/10.1023/A:1026504325010

SHANG, S.; SEDDON, P.B. (2000). A comprehensive framework for classifying the benefits of ERP systems. USA: Americas Conference on Information Systems AMCIS.

STADTLER, H.; KILGER, C. (2008). Supply Chain Management and advanced planning. New York: Springer. http://dx.doi.org/10.1007/978-3-540-74512-9

STETZER, A.; MORGESON, F.P. (1997). Organizational climate and ineffectiveness: Evidence from 25 outdoor work crew divisions, Journal Of Quality Management, 2(2): 251-265. http://dx.doi.org/10.1016/S1084-8568(97)90006-7

STRATMAN, J.K.; ROTH, A.V. (2002). Enterprise Resource Planning (ERP) competence constructs: Two-stage multi-item scale development and validation. Decision Sciences, 33(4): 601-628. http://dx.doi.org/10.1111/j.1540-5915.2002.tb01658.x

SU, Y.; YANG, C. (2010). Why are Enterprise Resource Planning systems indispensable to supply chain management? European Journal of Operational Research, 203(1): 81-94. http://dx.doi.org/10.1016/j.ejor.2009.07.003

SUN, H. (2011). A structural analysis of Quality Management practices in Hong Kong-based manufacturing companies. International Journal of Management, 28(3): 694-703.

SUPPIAH, V.; SANDHU, M.S. (2011). Organizational culture's influence on tacit knowledge sharing behaviour. Journal of Knowledge Management, 15(3): 462-477. http://dx.doi.org/10.1108/13673271111137439

SURESHCHANDAR, G.S.; RAJEMDRAN, C.; ANANTHARAMAN, R.N. (2003). The influence of Total Quality service age on quality and operational performance. Total Quality Management \& Business Excellence, 14(9): 1033-1052. http://dx.doi.org/10.1080/1478336032000090941

UGBORO, I.O.; OBENG, K. (2000). Top management leadership, employee empowerment, job satisfaction, and customer satisfaction in TQM organizations: An empirical study. Journal of Quality Management, 5(2): 247-272. http://dx.doi.org/10.1016/S1084-8568(01)00023-2

WHITE, G. (1996). A survey and taxonomy of strategy-related performance measures for manufacturing. International Journal of Operations \& Production Management, 16(3): 4261. http://dx.doi.org/10.1108/01443579610110486 
WIDODO, A. (2011). Building strategy quality. International Journal of Business and Management, 6(8): 180-192. http://dx.doi.org/10.5539/ijbm.v6n8p180

YUSUF, Y.; GUNASEKARAN, A.; ABTHORPE, M.S. (2004). Enterprise information systems project implementation: A case study of ERP in Rolls-Royce. International Journal of Production Economics, 87(3): 251-266. http://dx.doi.org/10.1016/j.ijpe.2003.10.004

ZEITZ, G.; JOHANNESSON, R.; RITCHIE, J.E. (1997). An employee survey measuring Total Quality Management practices and culture. Group and Organization Management, 22(4): 414-444. http://dx.doi.org/10.1177/1059601197224002

\section{Apéndice 1}

\section{Escala de medición de la cultura/orientación hacia la calidad (Jayaram, Ahire \& Dreyfus, 2010)}

Valore las siguientes afirmaciones desde $1=$ Total desacuerdo a $7=$ Total conformidad:

\section{Compromiso de la alta dirección}

CALT1. Los directivos promueven la utilización de herramientas relacionadas con la Gestión de la Calidad en los procesos productivos de la empresa

CALT2. Los directivos han recibido una formación adecuada en relación con la utilización de dichas herramientas

CALT3. La empresa provee de los recursos financieros y laborales, así como del tiempo necesarios para mejorar la calidad del producto

CALT4. La empresa provee de los recursos financieros y laborales, así como del tiempo necesarios para mejorar la calidad de los procesos

CALT5. Los directivos tiene entre sus responsabilidades la consecución de objetivos relacionados con la calidad

\section{Enfoque hacia el cliente}

ENFC1. Las reclamaciones de los clientes relacionadas con la calidad de los productos se dirigen a la persona adecuada que es capaz de dar una rápida respuesta a las mismas

ENFC2. La empresa dispone de un proceso sistemático para que las reclamaciones de los clientes se trasladen a futuras mejoras o novedades en la cartera de productos

ENFC3. El personal de atención al cliente está aleccionado para resolver rápidamente las reclamaciones que se les presenten

ENFC4. La empresa dispone de medidas objetivas de la satisfacción del cliente

ENFC5. Los indicadores seleccionados para medir la satisfacción del cliente son fiables

ENFC6. La empresa realiza comparaciones entre los indicadores de satisfacción del cliente propios y los de la competencia

\section{Confianza}

CONF1. La mayoría de los empleados considera que la empresa es rigurosa en el proceso de regulación de la calidad de sus productos

CONF2. Los responsables de los departamentos frecuentemente contrastan ideas con los empleados acerca de aspectos relacionados con la calidad de los productos

CONF3. La dirección y el resto de trabajadores contrastan abiertamente sus ideas acerca de aspectos relacionados con la calidad de los productos de la empresa

CONF4. La dirección y el resto de trabajadores muestran mutua confianza 
Escala de medición de los beneficios derivados de la implantación de un sistema de planificación avanzada de recursos ERP (Enterprise Resource Planning) (Su \& Yang, 2010)

Valore las siguientes afirmaciones desde $1=$ Total desacuerdo a $7=$ Total conformidad:

\section{Beneficios de tipo operativo}

El sistema de planificación avanzada de recursos ERP permite:

EROP1. Mejorar el control de los gastos operativos, disminuyendo de este modo los costes operativos en su conjunto

EROP2. Acortar el ciclo de vida de los productos, en especial cuando existe una amplia gama de los mismos, reduciendo de este modo el inventario

EROP3. Incrementar la productividad al involucrar a los consultores del sistema en la formación del personal dedicado a las tareas operativas

EROP4. Mejorar la gestión y el control de la calidad a nivel operativo

EROP5. Satisfacer proactivamente las necesidades del cliente, haciendo de este modo que la relación sea más eficiente

EROP6. Reducir el tiempo y los errores de los procesos que se desarrollan en el día a día de la empresa

\section{Beneficios de tipo directivo}

El sistema de planificación avanzada de recursos ERP permite:

ERDI1. Adaptar los productos para satisfacer más específicamente las necesidades de los consumidores, mejorando la gestión de los recursos de cara a conseguir una mayor personalización

ERDI2. Conseguir que sea más efectivo el proceso de toma de decisiones por parte de los trabajadores

ERDI3. Reducir costes, incrementar los ingresos y el valor de mercado de la empresa

ERDI4. Gestionar adecuadamente tanto las relaciones establecidas entre los diversos grupos de la empresa como las relaciones con grupos externos

ERDI5. Generar un funcionamiento más adecuado de la cadena de suministro de forma que se satisfagan mejor las necesidades de los consumidores

ERDI6. Mejorar la gestión y el control de la calidad a nivel directivo

\section{Beneficios estratégicos}

El sistema de planificación avanzada de recursos ERP permite:

ERES1. Facilitar el crecimiento de negocio

ERES2. Apoyar las alianzas en las que la empresa está inmersa

ERES3. Desarrollar innovaciones de negocio, permitiendo asimilar de forma rutinaria los cambios radicales

ERES4. Generar liderazgo en costes derivado del menor coste de seguimiento de inventario y de los menores costes laborales

ERES5. Originar diferenciación y personalización de producto

ERES6. Establecer vínculos externos que permitan mantener una mayor conexión con proveedores y clientes

\section{Beneficios derivados de la infraestructura de tecnologías de la información}

El sistema de planificación avanzada de recursos ERP permite:

ERTI1. Incrementar la flexibilidad de negocio frente a cambios presentes y futuros

ERTI2. Reducir los costes de mantenimiento que tenían los antiguos sistemas de gestión

ERTI3. Incrementar la capacidad determinada por la nueva infraestructura de tecnologías de la información

ERTI4. Integrar y controlar en tiempo real la información disponible

ERTI5. Estandarizar procesos desarrollados en diferentes localizaciones

ERTI6. Disponer de una interfaz sencilla unificando diferentes sistemas en uno sólo

\section{Beneficios organizativos}

El sistema de planificación avanzada de recursos ERP permite:

EROR1. Cambiar los procesos de dirección ampliándolos y expandiendo el horizonte de 
actuación de la empresa

EROR2. Facilitar el aprendizaje organizacional y la formación interna a través de la mayor información disponible

EROR3. Formar en habilidades relacionadas con el proceso de toma de decisiones generando confianza en los trabajadores a través del sentimiento de ser responsables de su propio trabajo

EROR4. Generar una visión común y general de la empresa

EROR5. Mejorar la moral y satisfacción de los trabajadores

EROR6. Formar en aspectos relacionados con la toma de decisiones en el contexto de un comportamiento adecuado a los intereses generales

(C) Intangible Capital, 2013 (www.intangiblecapital.org)

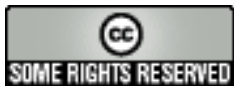

El artículo está con Reconocimiento-NoComercial 3.0 de Creative Commons. Puede copiarlo, distribuirlo y comunicarlo públicamente siempre que cite a su autor y a Intangible Capital. No lo utilice para fines comerciales. La licencia completa se puede consultar en http://creativecommons.org/licenses/by-nc/3.0/es/ 